\title{
LA FACULTAD DE CIENCIAS AGRARIAS Y EL VÍNCULO ESTABLECIDO CON LA COMUNIDAD EDUCATIVA
}

\section{Agrarian Sciences Faculty and its relationship with the educational community}

\author{
Sokolowski, Ana C. ${ }^{1}$; Seif, Juan M. ${ }^{1}$ \\ ${ }^{1}$ Facultad de Ciencias Agrarias, Universidad Nacional de Lomas de Zamora. soko576@hotmail.com
}

\begin{abstract}
RESUMEN
Desde los primeros años escolares se observa un alto índice de abandono, a lo que en la enseñanza media y secundaria se le suma repitencia, desgranamiento, menor promoción efectiva, sobreedad, menor motivación, lo que puede favorecer la deserción de estudiantes en la universidad. En este contexto, la Universidad debe salir y abrir las puertas a la comunidad. En este sentido, el objetivo del trabajo fue estudiar el vínculo generado entre la universidad y la comunidad educativa a través el Proyecto de Extensión Universitaria "La Facultad de Ciencias Agrarias extiende su mano a la comunidad que le dio origen y le ayudó a crecer" de la Facultad de Ciencias Agrarias (FCA) de la Universidad Nacional de Lomas de Zamora (UNLZ). La idea inicial se basó en generar mayor matrícula en la FCA. Sin embargo, esto no era suficiente pues la problemática excedía el mero hecho de convencer a los estudiantes a seguir sus estudios en esta institución. Para motivar e incrementar el vínculo de la comunidad con la universidad se pusieron en juego numerosas actividades, entre las que podemos mencionar visitas a colegios, visitas guiadas a la FCA, participación en Expos Universitarias, prácticas laborales y otras. Generar espacios de intercambio entre la universidad y la comunidad educativa establece vínculos más estrechos. Este vínculo permite que los estudiantes secundarios se relacionen con instituciones superiores y pierdan, el miedo a seguir una carrera universitaria o terciaria. Asimismo, realizar estas actividades de articulación entre enseñanza media y facultad como herramienta para la inclusión de los estudiantes.
\end{abstract}

Palabras Clave: relación, universidad, educación, inclusión, vínculo.

\begin{abstract}
From early school years, a high dropout rate is observed. Apart from that, in mid- and secondary school it is evidenced that repetition of school year, curtail shrinking, low rate of subject promotion, students over-age and low motivation might increase the drop out phenomenon at University level. In this context, the University must open wide to the community. In this sense, the aim of this work was to study the existing relationship between Lomas de Zamora National University (LZNU) and the school board through the University Extension Project: "The Agrarian Sciences Faculty (ASF) opens up to the Educational Community it has originally helped to develop". The initial idea was based on increasing students' enrollment at ASF. However, this was not enough as the problem exceeded the fact of fostering students' retention at this institution. In order to motivate and promote the bond between the community and the university, numerous activities were carried out, for instance: school visits, guided visits at ASF, Career Fairs and internships, among others. Generating exchanges between the university and the educational community forged closer links among them. This connection allows secondary school students to experience what university life is like so as not to be afraid to start College or University Education. Morover, carrying out articulation activities between highschool and University is an integration strategy that helps students.
\end{abstract}

Key words: relationship, univer sity, education, inclusion, connection.

Recibido: 20/may/2020. Aceptado: 24/jun/2020 
Sokolowski, A.C. y Seif, J.M.: Vinculo Facultad de Ciencias Agrarias- Comunidad Educativa.

\section{INTRODUCCIÓN}

Desde la escuela primaria se observa un alto índice de abandono, a lo que en la enseñanza media se le suma repitencia, desgranamiento, menor promoción efectiva, sobreedad, menor motivación y mayor matrícula en escuelas para adultos. Esto puede favorecer, en el futuro, la deserción de los estudiantes universitarios por lo que hay muchos estudios y propuestas de mejora (Mendoza Rojas, 2006; González, 2006). La brecha entre los modelos pedagógicos del sistema educativo puede imposibilitar a un gran número de estudiantes a adaptarse a los estudios superiores, los que quedan en alto riesgo de fracaso. También se observa un desgranamiento desde el inicio de las carreras. Sin embargo, tanto la retención como la deserción en el primer año están influenciadas por múltiples variables, personales e institucionales, que condicionan la evolución académica. Entre los aspectos personales que afectan a la retención, puede mencionarse que muchos ingresantes tienen escasa información acerca de lo que significa ser un estudiante universitario (De la Sota et al., 2010).

Las universidades forman parte de las instituciones sociales, que juegan un papel fundamental en la transformación de las sociedades (Castillo Cabeza et al., 2016). La extensión universitaria cumple varios roles fundamentales: formación continua de la propia comunidad universitaria en su conjunto, el intercambio de saberes y la divulgación científica. Por lo tanto, tiene un papel fundamental en la transformación social y el desarrollo comunitario. En este contexto, la Universidad debe salir y formar parte, pero también es importante que diversos sectores de la sociedad puedan entrar y conocer a la Universidad por dentro. Esta vinculación con el medio es enriquecida si dentro de la Institución, diferentes áreas se integran como en un proyecto común y trabajan articuladamente (Bünzli \& Barral, 2016).

Todo apunta a llevar la propuesta académica de la facultad para facilitar el acceso de los estudiantes secundarios a la universidad y acortar la brecha que los separa de la enseñanza Universitaria. Sobre todo, contribuir en su decisión de continuar con sus estudios luego de culminar su último año. Existe diversidad de opiniones entre ellos en cuanto a si seguir o no estudiando una vez concluida esa etapa. Muchos inician una carrera universitaria sin dudarlo respaldados en sus ideas y apoyados por sus familias que son profesionales o aspiran a que sus hijos lo sean para asegurar, relativamente, un futuro mejor. La decisión de seguir estudiando depende en gran parte del entorno (escuela, barrio y familia) de cada estudiante (González \& Aguirre de Quevedo, 2010), quienes pueden favorecerla o generar un conflicto más en sus múltiples miedos (Cortada, 1991). Otros por miedo, por falta de acompañamiento o conocimiento y/o problemas económicos ven a la universidad como un mundo inaccesible, imposible y distante (Sokolowski \& Seif, 2015). El ingreso a la Universidad es para los estudiantes un hecho gratificante, pero a la vez estresante. Existen múltiples factores que se conjugan en ese momento y transforman la vida del joven universitario (Huaquín \& Loaiza, 2004; Tonconi Quispe, 2010). El encuentro de nuevos compañeros de estudios, nuevas normas de trabajo y convivencia, nuevos requerimientos en el aprendizaje, en muchos casos el desprendimiento del grupo familiar y el desarraigo, son algunos de ellos (Guevara \& Henríquez, 1997). Alumnos cuyos padres no habían completado la enseñanza secundaria tienen mayor probabilidad de desertar en relación con aquellos cuyos padres tienen estudios universitarios completos (Barrios et al., 2011; Centro de Estudios Mineduc, 2012). Estos aspectos desempeñan un papel decisivo en el rendimiento escolar, por la posición económica-cultural, por los estímulos que constantemente recibe el niño para el estudio, hacia el trabajo y por las expectativas futuras depositadas en él (Pérez Serrano, 1981).

La articulación entre la Universidad y el Nivel Secundario es una temática abordada actualmente por las políticas educativas y está presente en la agenda del gobierno nacional para resolver algunas problemáticas y afrontar, en parte, al diagnóstico que muestra al sistema educativo como un conglomerado de instituciones separadas y aisladas entre sí.

En este sentido, el objetivo del presente trabajo fue estudiar el vínculo generado entre la universidad y la comunidad educativa, a través del proyecto de extensión "Agrarias en las escuelas" perteneciente a la Facultad de Ciencias Agrarias de la Universidad Nacional de Lomas de Zamora.

\section{METODOLOGÍA Y RESULTADOS OBTENIDOS}

A nivel educativo se observa un incremento del éxodo de los estudiantes secundarios del sistema educativo y el bajo ingreso a la educación superior. Esta problemática detectada fue el motor inspirador, desde el año 
2013, del Proyecto de Extensión Universitaria (PEU): "La Facultad de Ciencias Agrarias extiende su mano a la comunidad que le dio origen y la ayudó a crecer" llamado también "Agrarias en las Escuelas" perteneciente a la Facultad de Ciencias Agrarias de la Universidad Nacional de Lomas de Zamora (FCA-UNLZ) viene desarrollando diversas actividades de vinculación con el área de educación.

A partir de encuestas realizadas desde 2013 a ingresantes de la FCA-UNLZ se determinó que la mayoría de los mismos, en promedio el $80 \%$, provenía de distritos cercanos (menor a $35 \mathrm{~km}$ ). Por esto, se priorizó la articulación entre la Facultad y los colegios de estos distritos. La idea inicial del proyecto se basó en generar mayor matrícula en la FCA. A poco de iniciar con las actividades en el territorio se tomó conciencia que esto no era suficiente pues la problemática excede el mero hecho de convencer a los estudiantes a seguir sus estudios en esta institución. Tras reuniones con asistentes sociales de Lomas de Zamora, directivos y profesores de escuelas de la zona se decidió ayudar al estudiante a participar en distintas tareas vinculantes para mejorar su posición en la decisión de continuar sus estudios y finalizar la escuela secundaria. Asimismo, este vínculo generado entre la escuela media y la facultad busca acortar la brecha que los separa de la universidad y contribuir en su decisión de continuar con sus estudios luego de culminar su último año.

A lo largo de este proyecto desde 2013 han participado un gran número de docentes y estudiantes de la facultad (más de 25 personas). Además, en vinculación con la Subsecretaría de Educación del Municipio de Lomas de Zamora (MLZ) por una reciprocidad de los Proyectos "Proyectando mi Futuro" de Orientación Vocacional, han participado Lic. Mónica Cagnoli, Lic. Juan Visconti y las demás instituciones con quienes hemos trabajado de forma conjunta (UAI, U.K., Instituto Saenz, Instituto Siembra, Armada Argentina, etc.). Además, se tuvo vinculación con el Municipio de Esteban Echeverría (Jefatura Distrital, Consejo Escolar y Oficina de Empleos).

Una de las preocupaciones más relevantes que manifiestan los docentes, es la escasa vinculación entre los diferentes niveles educativos. Numerosos autores destacan la importancia de la articulación entre la universidad y la comunidad educativa (Cámara et al., 2014; Hermida Vázquez et al., 2015 y Castillo Cabeza et al., 2016). En este sentido, desde este proyecto se realizaron numerosas actividades que permitieron la articulación entre estos dos niveles educativos. A continuación, se enumeran las actividades ejecutadas:

\section{a)- Visitas a Colegios.}

Se visitaron distintos colegios de la zona de forma particular o dentro del proyecto de orientación vocacional de la MLZ, donde se presentó la oferta académica y las distintas actividades, con entrega de folletería y la donación de semillas de árboles, arbustos o especies hortícolas (Figura 1).

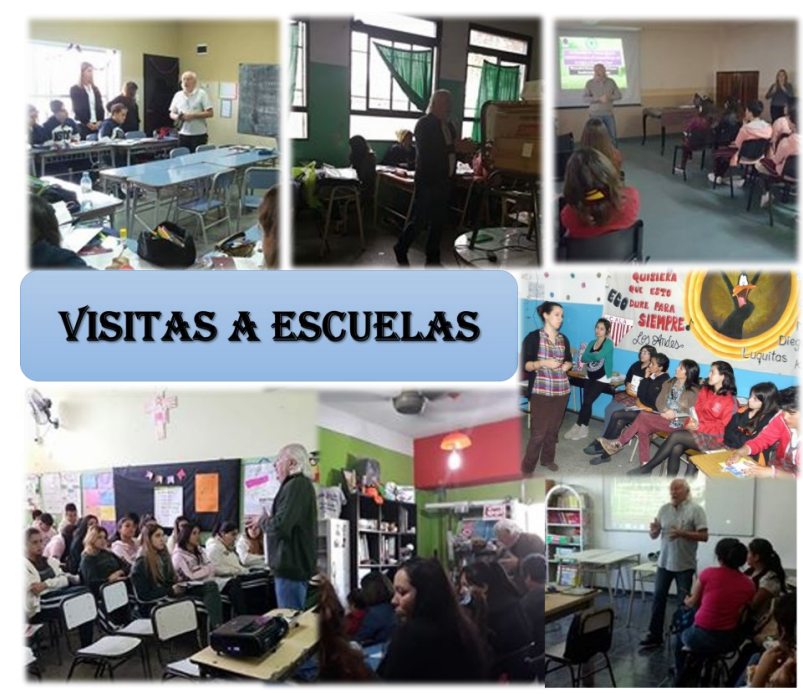

Figura 1. Visitas a colegios en las que ha participado el proyecto desde 2015 a 2019, donde se compartieron la oferta académica y actividades de la FCA. 


\section{b)- Visitas guiadas a la FCA.}

Las mismas consistieron en realizar una charla introductoria acerca de la facultad con entrega de folletería y bolsas de semillas y luego realizar una visita guiada por la sede vieja (Fair y Ruta 4) donde se ubican los dos museos, el laboratorio central, el invernáculo, la huerta, la estación meteorológica, los ensayos y los animales (vacas, terneros, conejos, cabras, aves, etc.). Se arma un circuito dinámico representativo de las actividades que se desarrollan. Finalmente, estudiantes, docentes y directivos culminan la jornada con una merienda o almuerzo al aire libre y, las escuelas realizan un cierre con una reflexión de los estudiantes. Aquí se invita a todo aquel nivel escolar que quiera participar (jardín de infantes, escuela especial, primaria, media o secundaria) y adultos. Los jóvenes cercanos a recibirse, tienen un primer contacto con la vida universitaria. Se destaca de estas actividades que, cada contacto generado es un iniciador de un efecto dominó que permite la toma de conocimiento de las carreras de la facultad y sobre las actividades que en ella se realizan (Figura 2).

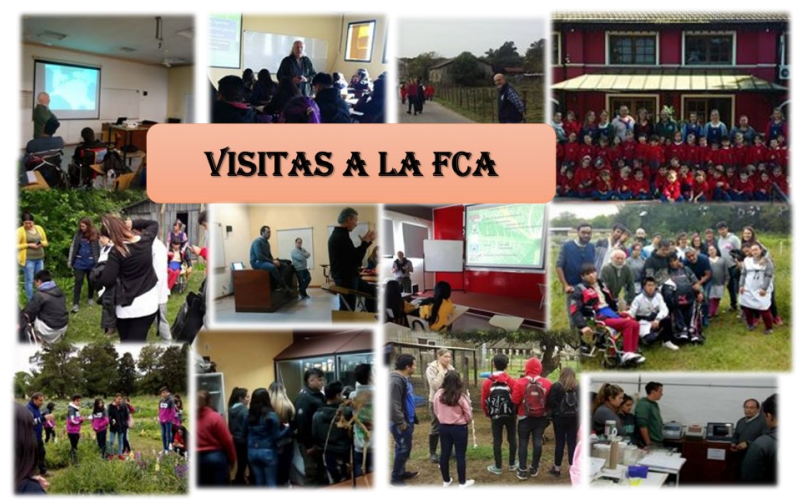

Figura 2. Visitas guiadas realizadas por var ias instituciones a la Facultad de Ciencias Agrarias, Univer sidad Nacional de Lomas de Zamora desde 2015 a 2019.

\section{c)-Expos Universitarias.}

Consistió en la participación en diversas expos que realizan distintas Instituciones/ colegios de distritos aledaños y no aledaños, donde se da a conocer un amplio abanico de posibilidades para los estudiantes y donde la facultad puede brindar la oferta académica y entablar conversación con muchísima gente donde se incluyen estudiantes, docentes, directivos, parientes y público en general (Figura 3). En estos eventos, se entrega folletería y bolsas de semillas de árboles o arbustos recolectados y confeccionados por el propio equipo de trabajo. Esto permite generar una conciencia de la naturaleza y la importancia de los árboles en el medio ambiente.

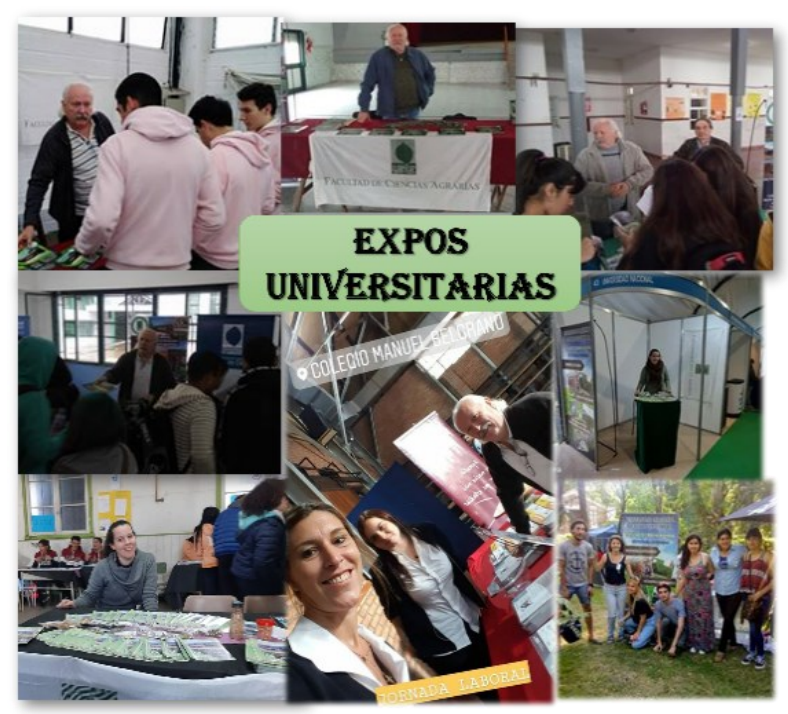

Figura 3. Fotos de varias Expos Univer sitarias en las que ha participado el proyecto desde 2015 a 2019. 


\section{d)-Prácticas Laborales:}

Consistió en la acreditación de horas prácticas con actividades en huerta, semillas, cunicultura, avicultura o con cursos del Centro de Educación Agraria Nº (Figura 4) para los colegios.

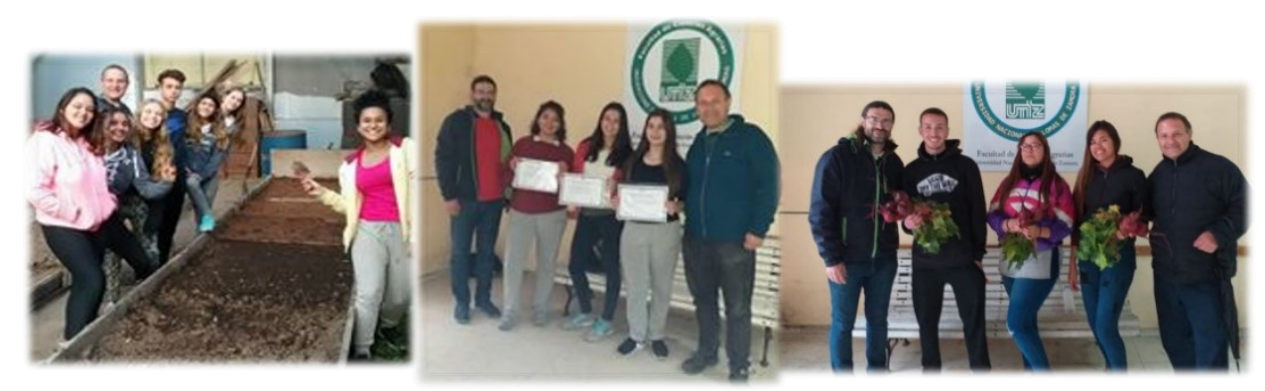

Figura 4. Prácticas profesionales realizadas en la facultad por alumnos del último año de colegio.

\section{e)- Otros:}

En este rubro se incluyen (Figura 5):

1) Expos de otras índoles: cierre de año de colegios agrotécnicos, Expos municipales, etc.

2)-Temáticas en las escuelas: Fueron Talleres teórico- prácticos en los Colegios, en temas como compost, huerta, vivero forestal, árboles nativos, hidroponía, suelos, producción animal (aves, conejos), control de plagas, etc. y otras charlas específicas. Entonces, más allá de informar en las escuelas sobre opciones de carreras, se prioriza el nexo con el estudiante y se fomenta su llegada a la Facultad y la inclinación por las carreras agropecuarias. Esta actividad se orienta a valorar la importancia del conocimiento agrotécnico para el cuidado y conservación del medio ambiente y la salud, según la temática que identifique cada taller (Companioni Turiño, 2006). Las Temáticas se adecuan en función de las posibilidades y necesidades de cada Institución y de quien dicta el taller. Las habilidades que gradualmente se forman, muestran los avances de los estudiantes respecto a sus conocimientos, valoraciones y conductas en relación con la agricultura. Esta actividad educativa brinda conocimientos y colabora con la generación de una vocación hacia las carreras agropecuarias que actualmente es insuficiente (Salas Batista, 2011).

3) Jornadas profesionales y diversos eventos organizados por la facultad (Jornadas de biología, jornadas científicas, etc.)

4) Actividades formales de extensión con escuelas (Día del ambiente, entrega semillas, calculadora de carbono, etc.).

También es importante destacar, dado los avances tecnológicos y la estrecha relación y hasta dependencia que tienen los jóvenes "nativos digitales" con las redes sociales (Del Barrio Fernández \& Ruiz Fernández, 2014), este proyecto de extensión tiene contacto y divulgación a través de Facebook e Instagram bajo el nombre "Agrarias en las escuelas".

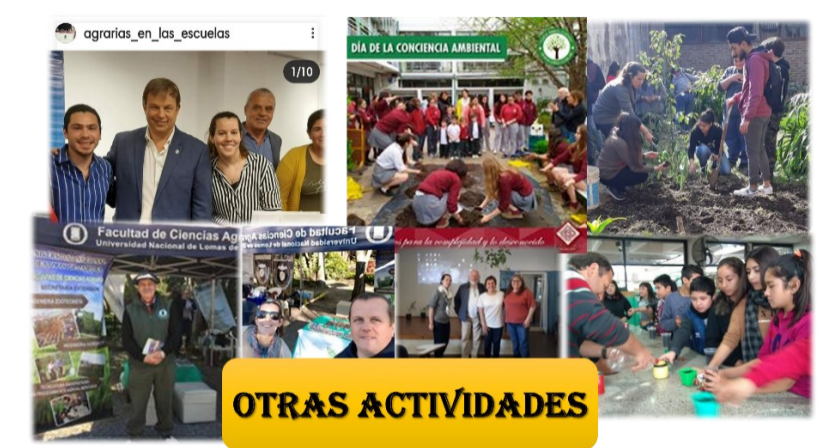

Figura 5. Otros eventos en los que ha participado el proyecto desde 2015 a 2019. 
Asimismo, se ha participado en notas del periódico Unión. Por último, a lo largo de estos años, se han presentado varios trabajos en revistas, congresos, jornadas o simposios de extensión y educación.

En todos los eventos se menciona la oferta académica, no solo por pertenecer a la institución y querer incrementar la matriculación, sino porque se los atrae hacia carreras que son más cercanas al ser humano desde tiempos remotos "La agricultura es la única fuente constante, cierta y enteramente pura de riqueza". Las actividades relacionadas al agro acompañan al ser humano desde que este abandonó sus hábitos de cazador y/o recolector. Para mejorar la preparación de los estudiantes y la selección profesional, debemos comprometernos con un sistema de actividades en el trabajo de orientación profesional-vocacional (Larrondo Chacón, 2012). En Cuba algunos directivos reconocen que propician una cultura alimentaria y el conocimiento de las raíces y tradiciones, situación actual de la agricultura en el mundo. Los directivos sienten que no logran, suficientemente, desarrollar los valores que potencia el trabajo con la tierra, por lo que se ven afectados los propósitos asociados a la formación vocacional y a la orientación profesional que ésta pudiera tener (Companioni Turiño, 2006). En este sentido, Scala et al. (2014) asocia la profesión con la vocación, a trabajar en lo que a uno le gusta, pero con responsabilidad.

En la Tabla 1 se resume la cantidad de eventos de cada actividad realizados, y la cantidad de estudiantes y docentes que han asistido y con los cuales se ha tenido contacto en los últimos cinco años del proyecto. Se aclara que las conferencias del Teatro Municipal de MLZ junto al municipio, que suman un número considerable se describen aparte dado que no se han realizado todos los años dependiendo del municipio.

Tabla 1. Cantidad de eventos, estudiantes, docentes, colegios y público en general que han asistido a cada actividad desde 2015 a 2019.

\begin{tabular}{llcccccc}
\hline & CANTIDADES & $\mathbf{2 0 1 5}$ & $\mathbf{2 0 1 6}$ & $\mathbf{2 0 1 7}$ & $\mathbf{2 0 1 8}$ & $\mathbf{2 0 1 9}$ & Total \\
\hline \multirow{2}{*}{$\begin{array}{l}\text { Visitas a } \\
\text { Colegios }\end{array}$} & Estudiantes & 1380 & 817 & 1145 & 953 & 6176 & 6176 \\
& Docentes o directivos & 53 & 54 & 93 & 72 & 395 & 395 \\
\hline \multirow{2}{*}{$\begin{array}{l}\text { Visitas } \\
\text { Guiadas a }\end{array}$} & Estudiantes e Infantes & 22 & 17 & 24 & 25 & 112 & 112 \\
\hline la FCA & Docentes o directivos & 66 & 51 & 53 & 90 & 417 & 417 \\
& Visitas & 12 & 14 & 17 & 28 & 124 & 124 \\
\hline \multirow{2}{*}{ Expos } & Estudiantes & 16815 & 18932 & 20150 & 13410 & 83681 & 83681 \\
& General & Sin dato & 7740 & 13040 & 15225 & 60055 & 60055 \\
& Expos & 15 & 26 & 23 & 23 & 106 & 106 \\
\hline Prácticas & Estudiantes & 5 & 6 & 7 & 2 & 45 & 45 \\
\hline \multirow{2}{*}{ Otros } & Colegios & 1 & 1 & 2 & 2 & 9 & 9 \\
\hline Conferencias & Estudiantes & 925 & 320 & 1215 & 566 & 5673 & 5673 \\
\hline & Docentes o directivos & Sin dato & 24 & 280 & 144 & 515 & 515 \\
\hline
\end{tabular}

En total el proyecto, considerando sólo este último período, ha tenido contacto con más de 160000 personas entre directivos, docentes, maestras/os, infantes, estudiantes de escuelas primarias, medias y secundarias públicas o privadas, escuela de adultos, padres, madres y público en general. Se destaca que, varios docentes, directivos o maestros/as se suman reiteradas veces año a año a alguna actividad, dado el gran vínculo generado. Desde el PEU de la FCA, a lo largo de estos últimos cinco años, se tuvo contacto con un total de 99000 estudiantes, 1327 docentes o directivos en más de 351 eventos, números significativos que señalan la presencia de la facultad en la comunidad.

Trabajos similares se han realizado por Larran et al. (2012) en la Facultad de Ciencias Agrarias y Forestales, Universidad Nacional de La Plata quienes realizaron talleres con directivos y docentes de escuelas secundarias, actividades áulicas teóricas y prácticas, salidas al medio, brindando información relacionada a las carreras, incumbencias profesionales, planes de estudio y forma đell ingreso, promoviendo la visita de alumnos a la Institución. 
La articulación entre los niveles educativos es un gran desafío que permite mejorar el desempeño académico inicial de los alumnos provenientes del nivel medio y lograr su retención y permanencia en las carreras. Lo que se busca con este trabajo es utilizar estrategias de articulación como una herramienta para hacer frente a la crisis del desgranamiento y la deserción en el nivel superior (Larran et al., 2012). Se recurrió a todos los recursos posibles para generar eventos y encuentros de vinculación con los estudiantes. Prueba de esto es la cantidad de personas con las cuales se llegó a relacionar desde el PEU.

A partir de charlas con docentes y directivos que se realizaron al finalizar cada actividad, principalmente en las visitas guiadas, se concluyó que éstas constituyeron un medio eficaz para establecer un vínculo entre la universidad y la comunidad circundante. Es una oportunidad de dialogar sobre la universidad, la vida universitaria y el ejercicio profesional donde las distintas instituciones señalan la importancia de que la actividad continúe en el tiempo, resultados que coinciden con lo encontrado por Bünzli \& Barral (2016). Agradecemos inmensamente a todos los que han participado a lo largo de los años y a aquellos que lo seguirán haciendo.

Pero más allá de números, se contempla el porcentaje de ingresantes que conocieron la FCA a través del proyecto que antes del PEU no existía. El PEU brinda además la posibilidad a estos estudiantes de acercarse a una facultad y ser partícipes de una actividad.

A partir de una encuesta realizada a los ingresantes se estudió cuál fue el verdadero impacto generado por estas actividades. Estas encuestas se realizaron por miembros del grupo que recorren los introductorios de febrero, los introductorios de marzo y los que se encuentran cursando el primer año y no pasaron por introductorios, para incluir a todos los ingresantes de cada año. La misma encuesta permite realizar un diagnóstico de la población ingresante (edad, colegio de procedencia, orientación, educación de los padres, tenías vocación por la carrera, cómo tomaste conocimiento de la existencia de la facultad y sus carreras). En este trabajo se tomó en cuenta la respuesta a la pregunta ¿cómo tomaste conocimiento de la existencia de la facultad?, que permite conocer la cantidad de ingresantes que cada año lo hacen gracias a este proyecto. Se observa un claro incremento desde el año 2016 a 2020 (Figura 6). Del año 2016 al año 2017 el incremento fue de 7,9 \%; del año 2017 al año 2018 fue de 7,35 \%; del año 2018 al año 2018 fue de 8,9 \% y el salto importante ya se observa de 2019 a 2020 siendo de $49 \%$. El incremento total del año 2016 al año 2020 gracias a la implementación de este proyecto fue del $87,3 \%$ con un elevado ajuste de la línea de tendencia.

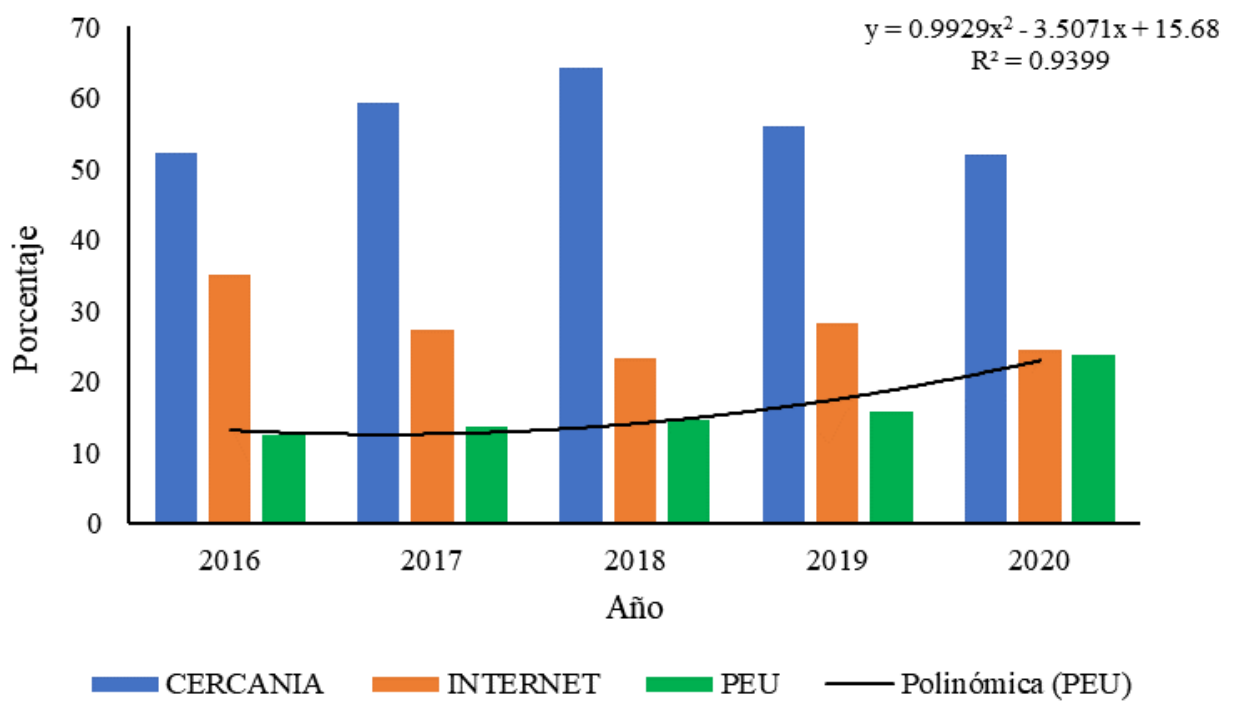

Figura 6. Respuesta a la pregunta ¿cómo tomaron conocimiento de la existencia de la facultad? de la encuesta realizada a la facultad. Todos los resultados han sido presentados de forma individual mediante informes ante la Secretaría de Extensión de la Facultad de Ciencias Agrarias de la Universidad Nacional de Lomas de Zamora. 


\section{CONSIDERACIONES FINALES}

Los espacios de intercambio generados entre la universidad y la comunidad educativa, producen vínculos más estrechos entre los diversos actores del sistema.

El vínculo entre la facultad y la escuela media permite que los estudiantes secundarios se relacionen con instituciones superiores y pierdan, por lo menos en parte, el miedo o el "no puedo" a seguir una carrera universitaria o terciaria. Es importante trabajar en eventos que vinculen estudiantes de todas las edades, independientemente de la orientación que posean sus escuelas, incluyendo familiares y amigos quienes tomarán conocimiento de la existencia de la FCA, percibiendo que estudiar una carrera universitaria no es imposible. Asimismo, realizar estas actividades de articulación entre la enseñanza media y la facultad constituyen una herramienta que facilita la inclusión de los estudiantes.

Se debe conocer la población ingresante para implementar nuevas estrategias metodológicas acordes a cada situación particular. Aún con una oferta Terciaria y Universitaria creciente (en número y cercanía) es muy notorio el abandono, desgranamiento y elección de carreras con las que los estudiantes no tienen la más mínima afinidad. Cuestión que contribuye a la sobreedad, otra característica encontrada.

En un futuro, sería de mucha utilidad, poder contrastar estos datos con otras instituciones educativas y conocer si se mantienen estos índices o le son propios al conurbano bonaerense o a las carreras vinculadas con el sistema agropecuario.

\section{Agradecimientos}

Queremos agradecer a los docentes y alumnos que han participado en alguna actividad realizada a lo largo de estos años de proyecto. Entre ellos: Ing. Zoot. Jorge Calvo; Ing. Agr. Víctor Milicia; Ing. Agr. Alberto De Magistris; Ing. Agr. Patricia N. Giola; Ing. Zoot. Néstor Urretabizkaya; Ing. Zoot. César López; todos los Integrantes del "Módulo Cabras" (Gloria Lynch, Laura Simonetti, Mercedes Mc Cormick, Mercedes Guibaudi), integrantes de varias cátedras (Forrajicultura, Mejoramiento Genético, Zoología, entre otros) no mencionados anteriormente. También al T.G.U. Ángel Sorba; al Ing.Agr. Diego Arakaki; al Ing. Agr. Diego Pérez; al Ing. Agr. Javier Pezzi y algunos estudiantes entre los cuales podemos nombrar a la Srta. Valeria Ponce; Belén Tammaro, Eliana Del Río; Ezequiel García y Georgina Martínez.

\section{BIBLIOGRAFÍA}

Barrios, M.A.; Meneses, F. \& Paredes, R. (2011). Financial aid and university attrition in Chile. Manuscrito no publicado.

Bünzli, A \& Barral, G. (2016). Visitas guiadas a la FCA. VI Congreso Nacional y V Congreso Internacional de Enseñanza de las Ciencias Agropecuarias, Bs As. Comunicación corta.

Cámara, A.; Lucchese, M.; Novella, M.L.; Fernández, A.R.; Benítez, M.S. \& Theiler, G.R. (2014). Articulación Educativa entre la Universidad y la Secundaria abordada desde la Virología. Revista EX N`5. UNC. ISSN 2250_7272.

Castillo Cabeza, S.N.; Canchingre-Bone, L.A. \& Becerra-Quiñonez, W.V. (2016). Reflexiones sobre la universidad y la sociedad. Dom. Cien. 2 ( $\mathrm{N}^{\circ}$ Especial): 444-454.

Companioni Turiño, B. (2006). Propuesta pedagógica para desarrollar la actividad agrícola, en la modalidad de agricultura urbana, en las escuelas secundarias básicas.

Centro de Estudios MINEDUC. (2012). Deserción en la educación superior en Chile. Serie Evidencias N ${ }^{\circ}$ 9.

Del Barrio Fernández, Á. \& Ruiz Fernández, I. (2014). Los adolescentes y el uso de las redes sociales. International Journal of Developmental and Educational Psychology. INFAD Revista de Psicología 1 (3) : 571-576 https://doi.org/10.17060/ijodaep.2014.n1.v3.537

De la Sota, P.; Coll Cárdenas, F. \& Merino, G. (2010). Acceso y permanencia en una educación de calidad. Problemáticas de los alumnos en el ingreso a primer año, en la Facultad de Ciencias Veterinarias de La Plata. Congreso Iberoamericano de Educación. Metas 2021. Buenos Aires, Argentina. https:// www.adeepra.org.ar/congresos/Congreso\%20IBEROAMERICANO/ACCESO/ $\underline{\text { R1022 Poster_delaSota.pdf }}$ 
González, L.E. (2006). Repitencia y deserción universitaria en América Latina. Informe sobre la educación superior en América Latina y el Caribe. 2000-2005. La metamorfosis de la educación superior. Primera Edición. IESALC. Caracas. pp 156-168. https://www.uv.mx/dgdaie/files/2013/09/americalatina.pdf

González, A. \& Aguirre de Quevedo, L. (2010). Factor es que influyen en los ingresantes a la Facultad de Ciencias Agrarias - UNCA, para la elección de la carrera. Del aula al campo, el desafío cotidiano 2:781. Ed Universidad Nacional de Entre Ríos.

Guevara, M.H. \& Henríquez, M.G. (1997). La transición del estudiante univer sitario: entre la elección y la deserción. I Encuentro Nacional "La Universidad como Objeto de Investigación" CEA -UBA.

Hermida Vázquez, N.A.; López Rodríguez del Rey, M.M \& Díaz Vera, C.E. (2015). Las relaciones entre la universidad y la escuela: su contribución al aprendizaje en la formación del profesorado. UNIVERSIDAD Y SOCIEDAD. Revista multidisciplinar de la Universidad de Cienfuegos. 7(3): 32-39.

Huaquín, V. \& Loaiza, R. (2004). Exigencias académicas y estrés en las carreras de la Facultad de Medicina de la Universidad Austral de Chile. Estudios Pedagógicos 30: 39-59. https://dx.doi.org/10.4067/ $\underline{\mathrm{S} 0718-07052004000100003}$

Larran, S.; Abramoff, C. \& Lampugnani, G. (2012). Un espacio inter disciplinario para la articulación nivel medio universidad. IV Congreso Nacional y III Congreso Internacional de Enseñanza de las Ciencias Agropecuarias, La Plata. Pags. 109-120

Larrondo Chacón, D. (2012). Actividades para la orientación profesional hacia las carreras agropecuarias. http://www.monografias.com/trabajos94/actividades-orientacion-profesional

Mendoza Rojas, J. (2006). Consolidación y Avance de la Educación Superior en México. Elementos de Diagnóstico y Propuestas. México. Editorial: Asociación Nacional de Universidades e Instituciones de Educación Superior (ANUIES). 275. ISBN: 970-704-099-8.

Pérez Serrano, G. (1981). Origen social y rendimiento escolar. Centro de Investigaciones Sociológicas (Madrid), Colección Monografías N44: 305 pp.

Salas Batista, N. (2011). La formación vocacional agropecuaria en el séptimo grado de la ESBEC Leonel Martí Corona. Edición Electrónica. www.eumed.net/libros/2011a/930/

Scala, E.; Mc Cormick, M. \& Scala, C. (2014). Las representaciones sociales de alumnos de primer año de la Facultad de Ciencias Agrarias, UNLZ: ¿Por qué eligen la carrera? ¿Qué significa ser ingeniero? $\mathrm{Y}$ ¿en qué suponen se puede trabajar?. Presentado en el V Congreso Nacional y IV Congreso Internacional de Enseñanza de las Ciencias Agropecuarias. CABA. Actas en la Revista de Divulgación Técnica Agropecuaria, Agroindustrial y Agroambiental Facultad de Ciencias Agrarias. UNLZ. 1(3): 532542.

Sokolowski, A.C. \& Seif, J.M. (2015). Articulación escuela media-facultad: temáticas en talleres: Compostera, Huerta y vivero forestal. XI Jornada Nacional de Extensión Universitaria. III Jornadas Regionales Metropolitanas. UNLa. Lanús Bs. As.

Tonconi Quispe, J. (2010). Factor es que influyen en el Rendimiento Académico y la Deserción de los estudiantes de la Facultad de Ingeniería Económica de la Una-Puno, período 2009. Cuadernos de Educación y Desarrollo 2 (11). https://www.eumed.net/rev/ced/11/jtq.htm 